\title{
Verification of Equivalent-Results Methods
}

\author{
K. Rustan M. Leino and Peter Müller \\ Microsoft Research, Redmond, WA, USA \\ \{leino, mueller\}amicrosoft.com
}

\begin{abstract}
Methods that query the state of a data structure often return identical or equivalent values as long as the data structure does not change. Program verification depends on this fact, but it has been difficult to specify and verify such equivalent-results methods and their callers.

This paper presents an encoding from which one can determine equivalentresults methods to be deterministic modulo a user-defined equivalence relation. It also presents a technique for checking that a query method returns equivalent results and enforcing that the result depends only on a user-defined influence set.

The technique is general, for example it supports user-defined equivalence relations based on Equals methods and it supports query methods that return newly allocated objects. The paper also discusses the implementation of the technique in the context of the Spec\# static program verifier.
\end{abstract}

\section{Introduction}

Computer programs contain many methods that query the state of a data structure and return a value based on that state. As long as the data structure remains unchanged, one expects different invocations of the query method to produce equivalent return values. For methods returning scalar values, the return values are expected to be the same. For methods returning object references, the most interesting equivalences are reference equality and equivalence based on the Equals method.

A simple and common example of a query method is the Count method of a collection class, like List in Fig. 0, where for a given collection the method returns the number of elements stored in the collection. Obviously, one expects Count to return identical values when called twice on the same collection. Another example is shown in the Calendar class in Fig. 2, where invocations of the GetEarliestAppointment will yield equivalent results as long as the state of the calendar does not change. However, since GetEarliestAppointment returns a newly allocated object, the results will not be identical. Due to object-allocation, query methods cannot be expected to be deterministic. Nevertheless, their results are expected to be equivalent. Therefore, we shall refer to such query methods as equivalent-results methods.

Query methods (also called pure methods) are particularly important in assertion languages such as JML [16] or Spec\# [2] because they allow assertions to be expressed in an abstract, implementation-independent way. For instance, Count is used in the precondition of GetItem (Fig. 0) to refer to the number of elements in the list without revealing any implementation details. However, reasoning about assertions that contain query methods is difficult. The client program in Fig.11illustrates the problem. It uses a

S. Drossopoulou (Ed.): ESOP 2008, LNCS 4960, pp. 307-321 2008.

(C) Springer-Verlag Berlin Heidelberg 2008 


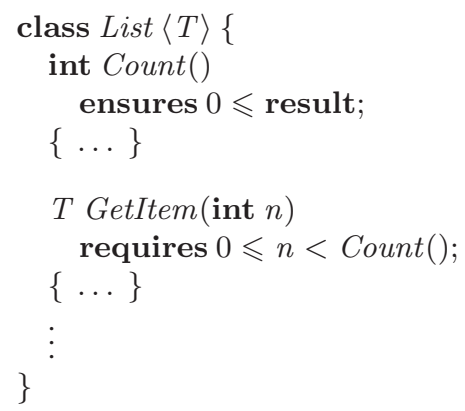

Fig. 0. A List class whose Count method returns the number of elements in a given list and whose GetItem method returns a requested element of the list. The postcondition of Count promises the return value to be non-negative, and the precondition of GetItem requires parameter $n$ to be less than the value returned by Count.

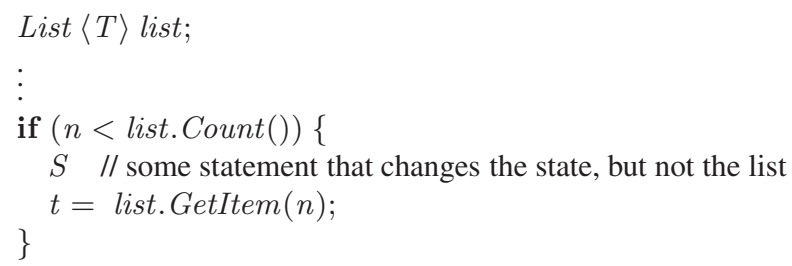

Fig. 1. A code fragment that uses the List class from Fig. 0 The if statement guards the invocation of GetItem to ensure that GetItem's precondition is met. To verify the correctness of this code, one needs to be able to determine that the two invocations of Count return the same value.

conditional statement to establish the precondition of GetItem. We assume that statement $S$ does not change the list structure. Therefore, we expect that the condition still holds when GetItem is called, that is, that the two calls to Count yield the same result. There are essentially three approaches for a program verifier to conclude this fact.

The first approach is to require that the postcondition of the query method is strong enough for a caller to determine exactly what value is returned. Typically, this can be achieved by having a postcondition of the form result $=E$. In our example, this postcondition would allow the verifier to compare the state affected by $S$ to the state read by $E$ to determine whether the two calls to Count return the same result. However, requiring such strong postconditions may entail a dramatic increase in the complexity of the specification. For Count, one would have to axiomatize mathematical lists and use that mathematical abstraction in the specification of the List class. We consider this burden too high, in particular for the verification of rather simple properties.

The second approach is to define the return value of the method to be a function of the program state. If the program state has not changed by the time the method is invoked again, this approach allows one to conclude the return value is the same as before. But this approach is too brittle, for two reasons. First, it treats state changes too coarsely. For example, statement $S$ in Fig. 1 may change the program state, but as long as it does not change the state of the list, we want to be able to conclude that the result 


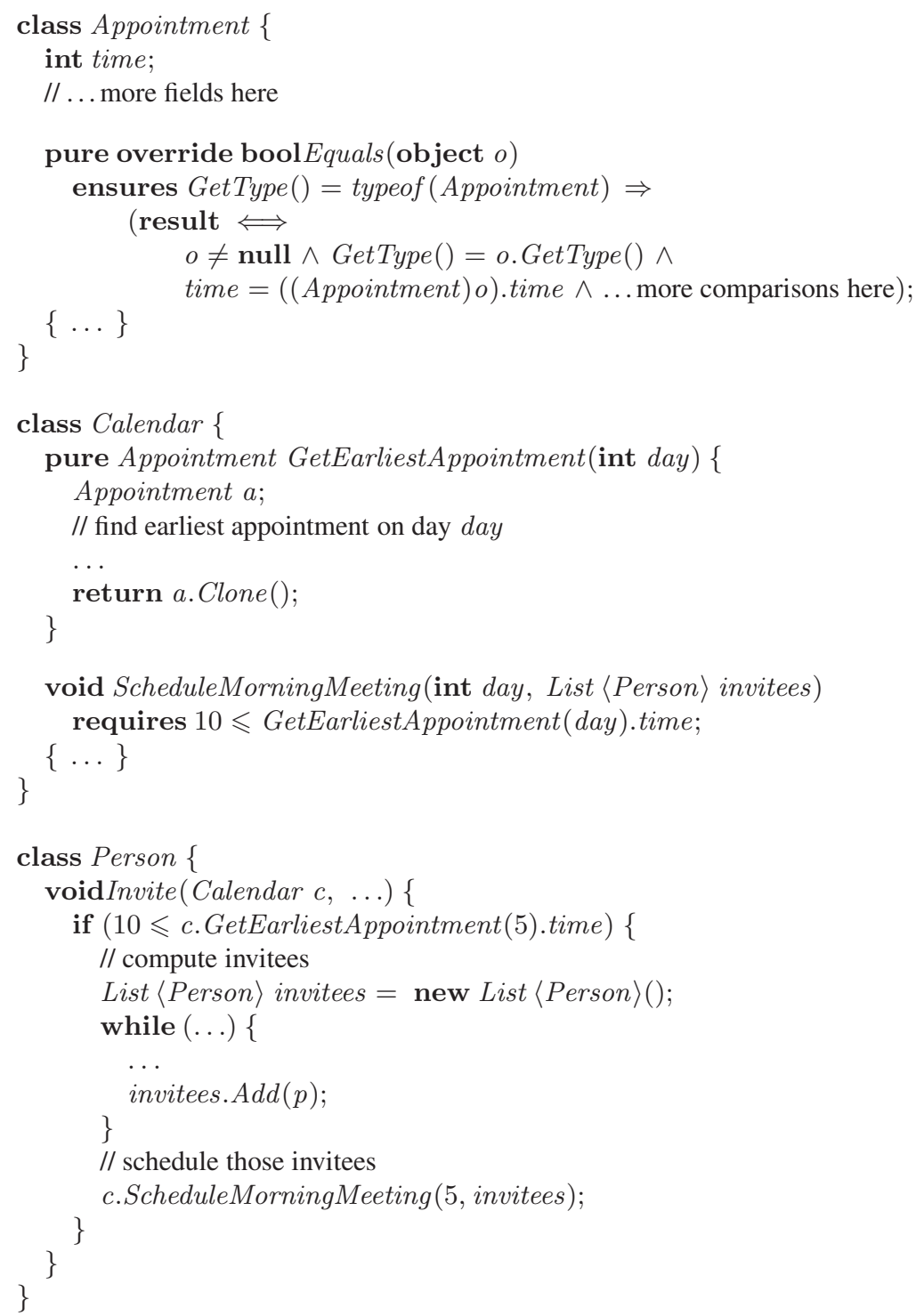

Fig. 2. A Calendar program whose GetEarliestAppointment method returns an equivalent value as long as the calendar does not change. The correctness of the code fragment at the bottom of the figure depends on that the call to GetEarliestAppointment in the precondition of ScheduleMorningMeeting returns a value that is equivalent to the one returned by the call to GetEarliestAppointment in the guard of the if statement.

of Count is unchanged. Second, this approach is too precise about the return value. For example, the object references returned by two calls to GetEarliestAppointment in Fig. 2 are not identical, yet the data they reference are equivalent. Queries that return newly allocated objects are very common, especially in JML's model classes [17]. 
The third approach is to require that all query methods used in specifications are equivalent-results methods whose results depend only on certain heap locations. We call this set of locations the influence set of a query method. With this approach, the code in Fig. 1 can be verified by showing that the locations modified by $S$ are not in the influence set of Count. From the equivalent-results property and the fact that Count returns an integer, we can conclude that the two calls to Count yield the same results.

Existing program verifiers such as the Spec\# static program verifier Boogie [1] and ESC/Java2 [15] apply the third approach. However, these systems do not enforce that query methods actually are equivalent-results methods and that their result actually depends only on the declared influence set. Blindly assuming these two properties is unsound. Checking the properties is not trivial, even for methods that return scalar values. For instance, GetHashCode is an equivalent-results method and should be permitted in assertions, but returning the hash code of a newly allocated object leads to nondeterminism and must be prevented.

In this paper, we present a simple technique to check that a query method is an equivalent-results method and that its result depends only on its parameters and the declared influence set. This technique supports user-defined equivalence relations based on, for instance, Equals methods. We use self-composition [3|21] to simulate two executions of the method body from start states that coincide in the influence set and to prove that the respective results are indeed equivalent. We also present axioms that enable reasoning about equivalent-results methods and argue why they are sound. Our technique is very general: it supports user-defined equivalence relations, it does not require a particular way of specifying influence sets, and it uses a relaxed notion of purity. In particular, implementations of query methods may use non-deterministic language features and algorithms, and may return newly allocated objects. We plan to implement our technique for pure methods in Boogie, but our results do not rely on the specifics of Spec\#. Therefore, they can be adopted by other program verifiers.

Outline. Section 1 provides the background on program verification that is needed in the rest of this paper. Section 2 presents an encoding of equivalent-results methods that enables the kind of reasoning discussed above. Section 3 explains our technique for checking the equivalence of results. Section 4 discusses the application of our technique to Spec\#. The remaining sections summarize related work and offer conclusions.

\section{Background on Program Verification}

In this section, we review details of program verification relevant to our paper. For a more comprehensive and tutorial account of this material, we refer to some recent Marktoberdorf lecture notes [20].

Architecture of Program Verifiers. To verify a program, the program's proof obligations (e.g., that preconditions are met) are encoded as logical formulas called verification conditions. The verification conditions are valid formulas if and only if the program is correct with respect to the properties being verified. Each verification condition is fed 


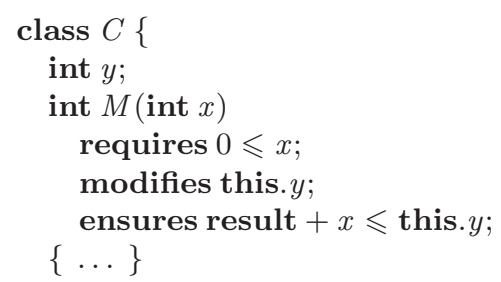

Fig. 3. An example class in the source language, showing an instance field $y$ and a method $M$ with a method specification

to a theorem prover, such as an SMT solver or an interactive proof assistant, which attempts to ascertain the validity of the formula or construct counterexample contexts that may reveal errors in the source program. As has been noted by several state-of-the-art verifiers, it is convenient to generate verification conditions in two steps: first encode the source program in an intermediate verification language, and then generate input for the theorem prover from the intermediate language [1125]. Since the second step concerns issues that are orthogonal to our focus in this paper, we look only at the first step. The notation we will use for the intermediate verification language is BoogiePL [111]. A BoogiePL program consists of a first-order logic theory, which in particular specifies the heap model of the source language, and an encoding of the source program. We explain these two parts in the following subsections.

Heap Model. We model the heap as a two-dimensional array that maps object identities and field names to values [24], so a field selection expression o.f is modeled as $\$ H e a p[o, f]$. By making the heap explicit, we correctly handle object aliases, as is well known [4]24]. In the encoding, we use a boolean field $\$$ alloc in each object to model whether or not the object has been allocated. The subtype relation is denoted by $<$.

For any set $S$ of locations (that is, of object-field pairs), we define a relation $\equiv_{S}$ that relates two heaps if they have the same values for all locations in $S$. More precisely:

$$
\left(\forall H, K, S \bullet\left(H \equiv_{S} K \Longleftrightarrow(\forall o, f \bullet(o, f) \in S \Rightarrow H[o, f]=K[o, f])\right)\right)
$$

Note that $\equiv_{S}$ is an equivalence relation: it is reflexive, symmetric, and transitive. If $H \equiv{ }_{S} K$, we say that $H$ and $K$ are equivalent modulo $S$.

We assume that pure methods do not modify the state of any object that is allocated in the pre-state of the method execution. This definition allows a pure method to allocate and modify new objects such as iterators [25]. More precisely, if $H 0$ and $H 1$ denote the heaps immediately before and after the call to a pure method, and $S$ is a set of locations of objects that are allocated in $H 0$, the following property holds:

$$
H 0 \equiv{ }_{S} H 1
$$

Encoding of Source Programs. Each source-language method is encoded as a procedure in the intermediate verification language. To understand the basic encoding, consider a method $M$ in a class $C$ with a field $y$, shown in Fig. 3 .

The specification of $M$ has a precondition that obligates the callers of $M$ to pass a non-negative argument value. In turn, the precondition lets the implementation of $M$ 


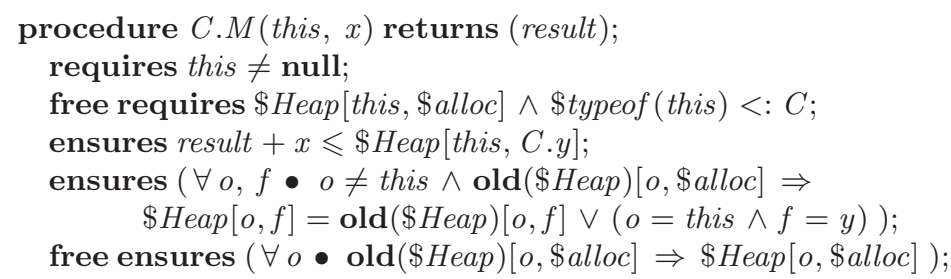

Fig. 4. A BoogiePL procedure declaration that encodes the signature and specification of the example method C.M

assume $x$ to be non-negative on entry. The specification also has a modifies clause and a postcondition that obligate the implementation to make sure that its return value, parameter $x$, and the $y$ field of the method's receiver object are related as specified, and to modify only this. $y$. A caller can assume these properties upon return of a call.

A representative encoding of $M$ as a BoogiePL procedure is shown in Fig. 4. The procedure declaration makes the implicit receiver parameter this explicit, and the anonymous return value is encoded as a named out-parameter. The types in BoogiePL are more coarse-grained than those in the source language, and for the purposes of this paper, they are only a distraction, so we omit them altogether. Three things are worth noting about the procedure specification.

First, method $M$ 's pre- and postconditions have direct analogs in the BoogiePL procedure, where the implicit dereferencing of the heap in a field selection expression is made explicit in the BoogiePL encoding.

Second, the method's modifies clause is encoded as a BoogiePL postcondition that dictates which locations in the heap are allowed to change. The latter says that for any non-null object $o$ allocated on entry to the method and for any field $f$, the heap at location $o . f$ is unchanged except possibly at location this. $y$.

Third, to verify a program, one often needs to know some properties that are guaranteed by the source language. For example, the static type of the receiver parameter of method $M$ is $C$ and the source-language type checker thus guarantees that the allocated type of the receiver is some subtype of $C$. The source language also guarantees that all object references in use by a program are allocated and (thanks to the fiction created by the garbage collector) remain allocated forever. To incorporate these guaranteed conditions in the encoding, BoogiePL conveniently offers free pre- and postconditions as part of a procedure declaration. Free preconditions are assumed on entry to a procedure implementation, but not checked at call sites, and analogously for free postconditions.

Proof Obligations and Soundness. Proving the correctness of a BoogiePL program amounts to statically verifying that the program does not abort due to a violated assertion (such as a precondition or postcondition). To do that, each assertion is turned into a proof obligation. One can then use an appropriate program logic to show that the assertions hold. For the proof, one may assume the conditions expressed as free preconditions, free postconditions, and explicit assume statements. The verification is sound if all of these assumptions actually hold. 


\section{Encoding of Equivalent-Results Methods}

Our idea is to define an equivalence class of return values for each equivalent-results method. We define the equivalence class via a programmer-defined similarity relation. Typical choices for the similarity relation are reference equality and the Equals method. Rather than letting the similarity relation be the equivalence relation, we define the equivalence class to be those values that are related by the similarity relation to a particular element, called the anchor element. This has the advantage that the similarity relation need not be symmetric and transitive, which in practice the Equals method often is not [26]. Another advantage is that using an anchor element allows us to state axioms that are handled more efficiently by the theorem prover.

In this section, we explain similarity relations, anchor elements, and the influence sets that define the dependencies of method results.

Similarity Relations. For a method $M$, we let $\mathcal{R}_{M}\left(H, r, H^{\prime}, r^{\prime}\right)$ denote $M$ 's similarity relation, relating $r$ whose state is evaluated in heap $H$ and $r^{\prime}$ whose state is evaluated in heap $H^{\prime}$. For example, if $\mathcal{R}_{M}$ denotes equality of scalar values or reference equality for object values, we have:

$$
\mathcal{R}_{M}\left(H, r, H^{\prime}, r^{\prime}\right) \Longleftrightarrow r=r^{\prime}
$$

and if $\mathcal{R}_{M}$ uses the Equals method, we have:

$$
\mathcal{R}_{M}\left(H, r, H^{\prime}, r^{\prime}\right) \Longleftrightarrow @ \operatorname{Equals}\left(H, r, H^{\prime}, r^{\prime}\right)
$$

where @Equals is a function automatically generated from the specification of Equals. Value $r$ is always a return value of the method; $r^{\prime}$ is either a return value, in which case $H=H^{\prime}$ or the anchor element, in which case $H^{\prime}$ is a special heap $\operatorname{AnchorHeap}_{M}(p)$ where we evaluate anchor elements. The similarity relation defines an equivalence class of values that are related to the anchor element.

For the Appointment.Equals method in Fig. 2 the following axiom is automatically generated for function @ Equals :

$$
\begin{aligned}
& (\forall H, \text { this }, K, o \bullet \\
& \text { this } \neq \text { null } \wedge \text { \$typeof }(\text { this })<: \text { Appointment } \wedge \text { \$typeof }(o)<\text { : Object } \Rightarrow \\
& (@ \text { Equals }(H, \text { this }, K, o) \Longleftrightarrow \\
& o \neq \text { null } \wedge \text { \$typeof }(\text { this })=\$ \text { typeof }(o) \wedge \\
& H[\text { this, time }]=K[\text { o, time }] \wedge \ldots \text { more comparisons here }))
\end{aligned}
$$

where, here and throughout, quantifications over $H$ and $K$ range over well-formed heaps. It is not the subject of our paper to describe how axioms for pure methods are described, but see our previous work with Ádám Darvas [10]9]; the difference is that here we use one heap argument for each of the two parameters to Equals.

Influence Sets. The influence set is a set of locations in the heap. Let $\mathcal{F}_{M}(H, p)$ denote the influence set of $M$ as computed for parameters $p$ in a heap $H$. Note that the computation of the influence set may depend on the heap. For example, consider 
a class Schedule with an Appointment field $a$. Suppose the influence set for some method applied to a schedule $s$ is given by the set of path expressions $\{$ s.a, s.a.time $\}$. Viewed in the intermediate-language notation, these path expressions denote the following object-field pairs: $(s, a),(\$ H e a p[s, a]$, time $)$.

We require every influence set to be self-protecting [14], which means that any two heaps equivalent modulo the influence set compute the influence set the same way:

$$
\left(\forall H, K, p \bullet H \equiv \overline{\mathcal{F}}_{M}(H, p) K \Rightarrow \mathcal{F}_{M}(H, p)=\mathcal{F}_{M}(K, p)\right)
$$

Self-protection can be enforced by requiring the set of path expressions that specify the influence set to be prefix closed: if it contains a path expression E.x.y, then it must also contain the path expression E.x. Therefore, the expression E.x.y denotes the same location in heaps $H$ and $K$.

The influence set specifies which parts of the program state are allowed to influence the return value. To a first order of approximation, the influence set is the read set or read effect of the method [6], but, technically, we actually allow methods to read any part of the state, as long as the values of things outside the influence set have no bearing on the return value.

Anchor Elements. The encoding of equivalent-results methods has to allow us to prove that two calls to an equivalent-results method $M$ return equivalent results if the two heaps before the calls are equivalent modulo the influence set of $M$. We reach this conclusion in two steps. First, we encode by an axiom that the anchor element remains the same as long as the program state indicated by the influence set does not change. Second, we encode by a free postcondition that the actual return value of $M$ is related to the anchor element by the similarity relation. Hence, the results of the two calls to $M$ are in the same equivalence class.

Step A: In our intermediate-language encoding, we introduce a function Anchor $_{M}$ that yields an anchor element for the equivalence class of the return values of $M$. We axiomatize Anchor $_{M}$ as follows:

$$
\left(\forall p, H, K \bullet H \equiv_{\mathcal{F}_{M}(H, p)} K \Rightarrow \operatorname{Anchor}_{M}(H, p)=\operatorname{Anchor}_{M}(K, p)\right)
$$

The axiom says that we pick the same anchor element whenever $M$ is invoked with the same arguments $p$ in two heaps $H$ and $K$ that are equivalent modulo $\mathcal{F}_{M}(H, p)$. In other words, the anchor element is a function of the program state projected onto the influence set.

Step B: We add to our encoding the following free postcondition:

$$
\text { free ensures } \mathcal{R}_{M}\left(\$ H e a p, \text { result, AnchorHeap } M(p), \operatorname{Anchor}_{M}(\$ H e a p, p)\right) \text {; }
$$

To make sure the anchor object always denotes the same equivalence class, we evaluate its state in a special, constant heap AnchorHeap $M$. We postpone until Section 3 how to justify this free postcondition. 


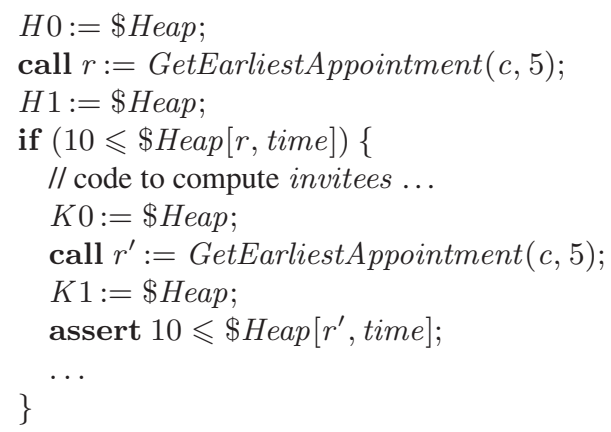

Fig. 5. A sketch of the code fragment from the bottom of Fig. 2 giving the names $H 0, H 1$, $K 0$, and $K 1$ to the intermediate values of the heap, and giving the names $r$ and $r^{\prime}$ to the return values of the two calls to GetEarliestAppointment. The assert statement at the end shows the condition that we want to prove.

Example. To prove the correctness of method Invite in Fig. 2] it suffices to show that the two invocations of GetEarliestAppointment return equivalent values. Recall, the second invocation takes place during the evaluation of the precondition of ScheduleMorningMeeting. Fig. 5] shows a BoogiePL encoding of that fragment. As illustrated by the assert statement in Fig. 5, we wish to prove that $H 1[r$, time $]$ equals $K 1\left[r^{\prime}\right.$, time $]$.

The influence set of GetEarliestAppointment contains the fields that make up the representation of the Calendar object. Let $H 0$ and $H 1$ denote the heaps immediately before and after the first call to GetEarliestAppointment, and let $K 0$ and $K 1$ denote the heaps immediately before and after the second call.

Since GetEarliestAppointment is pure, it does not change the values of any previously allocated locations (see condition (0) ), so $H 0$ and $H 1$ are equivalent modulo $\mathcal{F}(H 0, c, 5)$, and $K 0$ and $K 1$ are equivalent modulo $\mathcal{F}(K 0, c, 5)$ (we drop the subscript GetEarliestAppointment in this example). Assuming that the code that computes invitees has no effect on the values of the locations in the influence set, we also have that $H 1$ and $K 0$ are equivalent modulo $\mathcal{F}(H 1, c, 5)$. By self-protection (4), we know that the three influence sets are equal. Thus, we can conclude by transitivity:

$$
H 1 \equiv \mathcal{F}(H 1, c, 5) K 1
$$

By axiom (5) and equation (7), we conclude that the anchor elements for the two calls are the same:

$$
\operatorname{Anchor}(H 1, c, 5)=\operatorname{Anchor}(K 1, c, 5)
$$

Now let $r$ and $r^{\prime}$ denote (as indicated in Fig. 57 the values returned by the two calls to GetEarliestAppointment. The similarity relation is given by the Equals method. Thus, we conclude from postcondition (6):

@ Equals(H1, r, AnchorHeap (c,5), Anchor $(H 1, c, 5)) \quad$ and

@ Equals $\left(K 1, r^{\prime}\right.$, AnchorHeap $(c, 5)$, Anchor $\left.(K 1, c, 5)\right)$ 


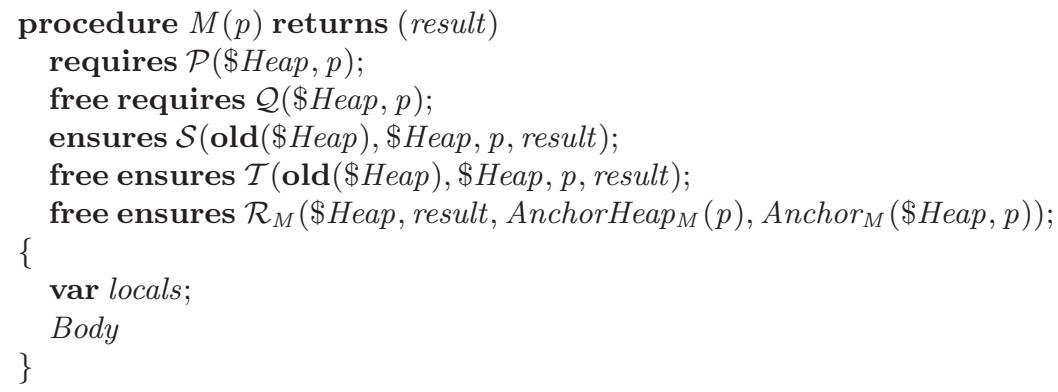

Fig. 6. A procedure in the intermediate verification language, illustrating the general form of the procedure into which the method translates

By axiom (3) and property (8), we have

$$
\begin{aligned}
& H 1[r, \text { time }]=\text { AnchorHeap }(c, 5)[\text { Anchor }(H 1, c, 5), \text { time }] \wedge \\
& K 1\left[r^{\prime}, \text { time }\right]=\text { AnchorHeap }(c, 5)[\text { Anchor }(H 1, c, 5), \text { time }]
\end{aligned}
$$

from which we conclude $H 1[r$, time $]=K 1\left[r^{\prime}\right.$, time $]$, as required to establish the precondition of the call to ScheduleMorningMeeting .

\section{Verifying Equivalence of Results}

As we mentioned in Section 1, soundness of a verification system comes down to justifying every assumption that the proof system allows a proof to make use of. In the previous section, we introduced three conditions that we used as assumptions in the proof. The first assumption is the axiom of self-protection (4). It can be justified by a syntactic check on the path expressions used to define the influence set. The second assumption is the axiom about Anchor $_{M}$ (5). It is justified on the basis that there exists a function Anchor $_{M}$ that satisfies the axiom, for example any constant function. The third assumption is the free postcondition (6). In this section, we present a proof technique based on self-composition that justifies this assumption.

Ordinarily, a method $M$ gives rise to a verification condition prescribed by a BoogiePL procedure implementation like procedure $M$ in Fig. 6, where $p$ denotes the in-parameters, $\mathcal{P}$ and $\mathcal{S}$ denote some checked pre- and postconditions, $\mathcal{Q}$ and $\mathcal{T}$ denote some free pre- and postconditions ( $c f$. Fig. 4), locals are local variables, and Body is the BoogiePL encoding of the implementation of method $M$.

For every equivalent-results method $M$, we will now prescribe a second BoogiePL procedure, whose validity will justify the free postcondition (6). The key idea is to execute the method body twice starting in states that agree on the values of the inparameters and all objects in the influence set. We then prove that the two executions yield equivalent results. This second procedure has the form shown by $M^{\prime}$ in Fig.7 7 and is described as follows:

- The body of $M^{\prime}$ starts off with $\$$ Heap, locals, and result set to arbitrary values, saves the value of $\$$ Heap in $\$$ oldHeap, and assumes the preconditions $\mathcal{P}$ and $\mathcal{Q}$. 


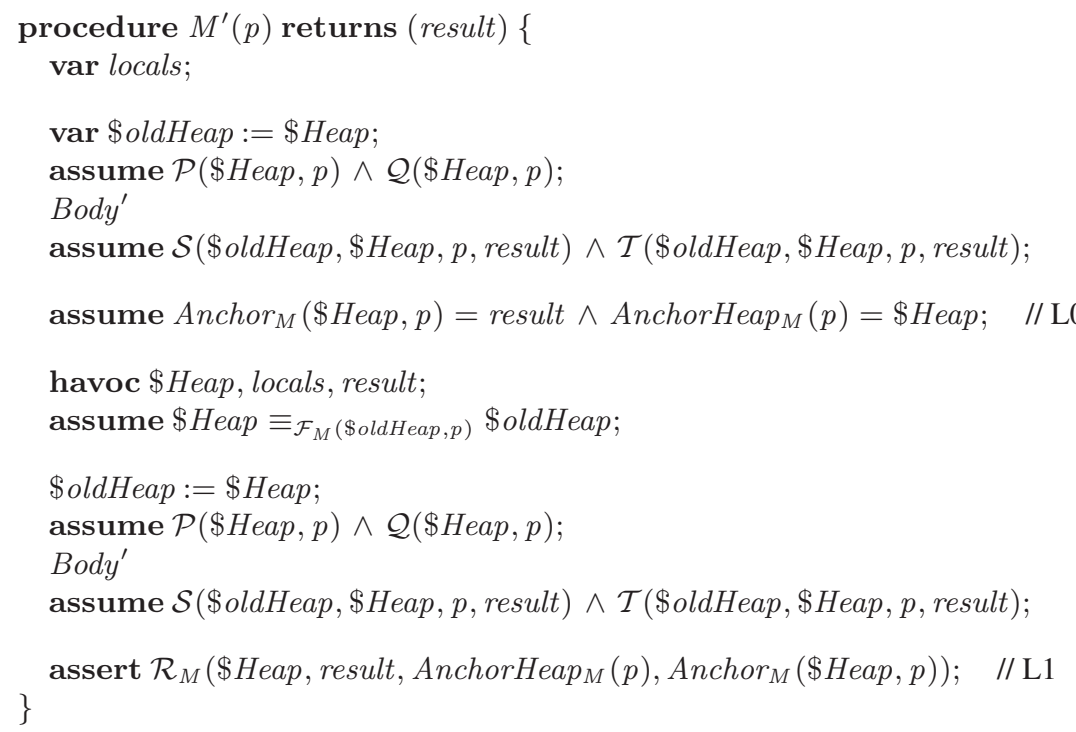

Fig. 7. A procedure that checks by assertion (L1) that $M$ satisfies its free postcondition 6

- It then performs Body', which is Body with occurrences of old $(\$ H e a p)$ replaced by $\$$ oldHeap and occurrences of assert statements (i.e., checked conditions) replaced by assume statements. These assume statements are justified by the fact that procedure $M$ already prescribes checks for them, so if the conditions do not hold, the program verifier will generate appropriate errors when attempting to verify $M$.

- Upon termination of Body', the postconditions $\mathcal{S}$ and $\mathcal{T}$ are assumed. Again, $\mathcal{S}$ can be assumed here because it is checked by $M$.

- We explain the assume statement (L0) below.

- Next, the code prepares for another execution of Body'. The second execution of $B o d y^{\prime}$ is to start in a state where all locations of the influence set have the same values as in the first execution. Thus, $\$$ Heap, locals, and result are set to arbitrary values (using a havoc statement) and the value of $\$$ Heap is constrained (using an assume statement) to be equivalent to $\$$ oldHeap modulo the influence set.

- The preconditions are assumed, Body' is executed a second time, and the postconditions are assumed.

- We explain the assert statement (L1) below.

The first half of $M^{\prime}$ culminates in assume statement (L0), which has the effect of defining Anchor $_{M}(\$ H e a p, p)$ and AnchorHeap $M(p)$ to be the result value and result heap of an arbitrary execution of the method (namely, the first execution of $B o d y^{\prime}$ ). In fact, by axiom (5), (L0) defines $\operatorname{Anchor}_{M}(\$ H e a p, p)$ for all heaps that are equivalent to $\$$ Heap modulo the influence set. The second half of $M^{\prime}$ checks that (6) is indeed a postcondition of the method for all those equivalent heaps.

With that, we have justified all the assumptions that our technique introduces, and thus we have established that our technique is sound. 


\section{Application to Spec\#}

In verifying Spec\# programs, we have run across scores of examples like the one in Fig. [0, where in Spec\# the Count method tends to be a property getter, which is a form of parameter-less method. By default, property getters are treated as pure methods that read only the ownership cone of the receiver object. The ownership cone of an object is the set of locations that make up the object's representation [7]. Previously, our best solution for dealing with this situation in the Spec\# program verifier was to introduce an axiom that says the return value of the method is a function of the ownership cone. But such an axiom is not sound if a pure method returns newly allocated object or values that are derived from such objects. Our technique in this paper gives a sound solution to the problem, and we intend to implement it. In this section, we describe some issues that pertain to the practical implementation of equivalent-results methods in Spec\#.

We intend to restrict the choices for $\mathcal{R}_{M}$ in Spec\# to support only the two choices (1) and (2). This will simplify the implementation while supporting the most common similarity relations. (The only other useful similarity we found puts all non-null references in one equivalence class.) To select between the two choices, we will introduce a default choice and a method annotation (a custom attribute) that can override the default.

For the influence set, we will only support the union of the ownership cones for some subset of the parameters. Ownership provides a form of abstraction, allowing one to specify influence sets without being specific about implementation details. There is already a notion of confined in Spec\# that says that a pure method reads the ownership cone of a parameter. Moreover, the Spec\# program verifier already has an encoding that lets one deduce, for valid objects, whether or not the ownership cone of the object has changed. The encoding is simply to inspect the object's ghost field snapshot [9]. An object is valid when its object invariant holds [19]. Since this is the precondition of almost all methods, we will not attempt to prove ownership cones to be the same other than via the snapshot field. Because of the snapshot encoding, we can write axiom (5) as:

$$
\begin{gathered}
(\forall p, H, K \bullet H[p, \text { valid }] \wedge K[p, \text { valid }] \wedge H[p, \text { snapshot }]=K[p, \text { snapshot }] \\
\left.\Rightarrow \operatorname{Anchor}_{M}(H, p)=\operatorname{Anchor}_{M}(K, p)\right)
\end{gathered}
$$

(We have abused notation slightly: by $H[p$, valid $]$ and $H[p$, snapshot $]$, we really mean to refer to the valid and snapshot fields of all the parameters in $p$ that contribute to the influence set, and likewise for $K$.) In fact, there is an alternative way to encode this property that is significantly more efficient for the SMT solver because it avoids quantification over pairs of heaps. The alternative encoding [9] introduces an uninterpreted function $A_{M}$ and uses it to more directly say that $\operatorname{Anchor}_{M}(H, p)$ is a function of $p$ and $H[p$, snapshot $]$ :

$$
\left(\forall p, H \bullet H[p, \text { valid }] \Rightarrow \operatorname{Anchor}_{M}(H, p)=A_{M}(p, H[p, \text { snapshot }])\right)
$$

With the restriction to influence sets based on ownership cones and our focus on reasoning about these via snapshots, axiom (4) becomes trivial, so we omit it. 


\section{Related Work}

The Java Modeling Language (JML) requires pure methods to be deterministic [18]. This requirement is not practical since pure methods often need to return newly allocated objects, which is illustrated by many pure methods in JML's model library [17]. Our notion of equivalent-results methods allows pure methods to return newly allocated objects. Since our axioms are based on a user-defined similarity relation such as an Equals method, determinism is not required for soundness.

The axiomatization of pure methods consists of two groups of axioms: methodspecific axioms that specify the behavior of each individual method and general axioms that describe common properties of all pure methods. Previous work by Darvas and Müller [10] focuses on the method-specific axioms, but does not discuss the general axioms that we provide in this paper. Their axiomatization is sound, but too weak for many interesting examples. Darvas and Leino [9] present general axioms that are used in the Spec\# verifier Boogie. Some of their work assumes that a pure method is deterministic and that its result depends only on a specified influence set, but these assumptions are not checked. Therefore, their axiomatization is unsound for pure methods that return newly allocated objects or whose result depends on locations outside the influence set. Our work eliminates both sources of unsoundness.

Jacobs developed SpecLeuven, a variant of Spec\# for multi-threaded programs. In his work [13], inspector methods are syntactically enforced to be deterministic, which is sound but overly restrictive. Influence sets are checked by an extension of the Boogie methodology [19], which requires an object to be unpacked before its state is read. Our verification technique based on self-composition does not require any particular methodology.

ESC/Java2 [15 8] also operates under the unchecked assumption that pure methods are deterministic, which is unsound if they are not. Moreover, since JML specifications typically do not declare an influence set, ESC/Java2 has but limited support for reasoning about the effect of a heap modification on the result of a pure method.

The influence sets we use in this paper are similar to read effects. However, read effects constrain the whole execution of a method, whereas our influence sets only constrain the method result. We allow methods to read arbitrary locations as long as the result depends only on the declared influence set. Clarke and Drossopoulou [6] show how to declare and check read effects in an ownership type system. We use selfcomposition to verify influence sets, which is in general more fine-grained than type checking and does not require a particular ownership scheme.

Self-composition has been applied to prove secure information flow [3 21]. In fact, proving that a method result depends only on a specified influence set can be seen as an instance of secure information flow, where the method result, the method parameters, and the locations in the influence set have a low security level and all other locations have a high security level. In addition to information flow, we use self-composition to prove that two executions of a method yield equivalent results.

Separation logic [22] provides a powerful and elegant way to reason about the effects of heap modifications. The effect of pure methods can be achieved by introducing abstract predicates [23]. The influence set of a pure method corresponds to the footprint of the predicate. The frame rule can be used to show that certain heap modifications do 
not affect the truth value of the abstract predicate. However, even if pure methods are not used in contracts, the correctness of some programs relies on the equivalent-results property. We believe that our verification technique is also applicable to separation logic in order to verify such programs.

\section{Conclusions}

In this paper, we introduced the notion of equivalent-results methods and explained their usefulness for program specification: equivalent-results methods are expressive, for instance, they may return newly-allocated objects, and they permit an axiomatization that is sound and strong enough to verify interesting programs. We showed that the equivalent-results property can be checked by an automatic program verifier using selfcomposition. Our technique is very flexible: it does not require a particular programming methodology, uses a relaxed notion of purity, and even handles non-deterministic language features and algorithms. As future work, we plan to implement our technique in the Spec\# verifier Boogie.

Acknowledgments. The idea of using self-composition was inspired by a discussion with Anindya Banerjee. We thank David Naumann and the anonymous reviewers for helpful comments, one of which led to a simplification of Fig. 7.

\section{References}

1. Barnett, M., Chang, B.-Y.E., DeLine, R., Jacobs, B., Leino, K.R.M.: Boogie: A Modular Reusable Verifier for Object-Oriented Programs. In: de Boer, F.S., Bonsangue, M.M., Graf, S., de Roever, W.-P. (eds.) FMCO 2005. LNCS, vol. 4111, pp. 364-387. Springer, Heidelberg (2006)

2. Barnett, M., Leino, K.R.M., Schulte, W.: The Spec\# Programming System: An Overview. In: Barthe, G., Burdy, L., Huisman, M., Lanet, J.-L., Muntean, T. (eds.) CASSIS 2004. LNCS, vol. 3362, pp. 49-69. Springer, Heidelberg (2005)

3. Barthe, G., D'Argenio, P.R., Rezk, T.: Secure information flow by self-composition. In: Computer Security Foundations (CSFW), pp. 100-114. IEEE Computer Society Press, Los Alamitos (2004)

4. Burstall, R.M.: Some techniques for proving correctness of programs which alter data structures. Machine Intelligence 7, 23-50 (1972)

5. Chatterjee, S., Lahiri, S.K., Qadeer, S., Rakamarić, Z.: A Reachability Predicate for Analyzing Low-Level Software. In: Grumberg, O., Huth, M. (eds.) TACAS 2007. LNCS, vol. 4424, pp. 19-33. Springer, Heidelberg (2007)

6. Clarke, D.G., Drossopoulou, S.: Ownership, encapsulation and the disjointness of type and effect. In: OOPSLA. SIGPLAN Notices, vol. 37(11), pp. 292-310. ACM Press, New York (2002)

7. Clarke, D.G., Potter, J.M., Noble, J.: Ownership types for flexible alias protection. In: OOPSLA. SIGPLAN Notices, vol. 33(10), pp. 48-64. ACM Press, New York (1998)

8. Cok, D.: Reasoning with specifications containing method calls and model fields. Journal of Object Technology 4(8), 77-103 (2005) 
9. Darvas, Á., Leino, K.R.M.: Practical Reasoning About Invocations and Implementations of Pure Methods. In: Dwyer, M.B., Lopes, A. (eds.) FASE 2007. LNCS, vol. 4422, pp. 336-351. Springer, Heidelberg (2007)

10. Darvas, Á., Müller, P.: Reasoning about method calls in interface specifications. Journal of Object Technology 5(5), 59-85 (2006)

11. DeLine, R., Leino, K.R.M.: BoogiePL: A typed procedural language for checking objectoriented programs. Technical Report MSR-TR-2005-70, Microsoft Research (March 2005)

12. Filliâtre, J.-C.: Why: a multi-language multi-prover verification tool. Research Report 1366, LRI, Université Paris Sud (March 2003)

13. Jacobs, B.: A Statically Verifiable Programming Model for Concurrent Object-Oriented Programs. PhD thesis, Katholieke Universiteit Leuven (2007)

14. Kassios, I.T.: Dynamic Frames: Support for Framing, Dependencies and Sharing Without Restrictions. In: Misra, J., Nipkow, T., Sekerinski, E. (eds.) FM 2006. LNCS, vol. 4085, pp. 268-283. Springer, Heidelberg (2006)

15. Kiniry, J.R., Cok, D.R.: ESC/Java2: Uniting ESC/Java and JML. In: Barthe, G., Burdy, L., Huisman, M., Lanet, J.-L., Muntean, T. (eds.) CASSIS 2004. LNCS, vol. 3362, pp. 108-128. Springer, Heidelberg (2005)

16. Leavens, G.T., Baker, A.L., Ruby, C.: JML: A notation for detailed design. In: Behavioral Specifications of Businesses and Systems, pp. 175-188. Kluwer Academic Publishers, Dordrecht (1999)

17. Leavens, G.T., Cheon, Y., Clifton, C., Ruby, C., Cok, D.R.: How the design of JML accommodates both runtime assertion checking and formal verification. Science of Computer Programming 55(1-3), 185-208 (2005)

18. Leavens, G.T., Poll, E., Clifton, C., Cheon, Y., Ruby, C., Cok, D., Müller, P., Kiniry, J.: JML reference manual. Dept. Comp. Sci., Iowa State University (2007),

http: / / www.jmlspecs.org

19. Leino, K.R.M., Müller, P.: Object Invariants in Dynamic Contexts. In: Odersky, M. (ed.) ECOOP 2004. LNCS, vol. 3086, pp. 491-516. Springer, Heidelberg (2004)

20. Leino, K.R.M., Schulte, W.: A verifying compiler for a multi-threaded object-oriented language. In: 2006 Marktoberdorf Summer School on Programming Methodology, Springer, Heidelberg (to appear, 2007), research.microsoft.com/ leino/papers.html

21. Naumann, D.A.: From Coupling Relations to Mated Invariants for Checking Information Flow. In: Gollmann, D., Meier, J., Sabelfeld, A. (eds.) ESORICS 2006. LNCS, vol. 4189, pp. 279-296. Springer, Heidelberg (2006)

22. O'Hearn, P.W., Yang, H., Reynolds, J.C.: Separation and information hiding. In: POPL, pp. 268-280 (2004)

23. Parkinson, M.J., Bierman, G.M.: Separation logic and abstraction. In: POPL, pp. 247-258. ACM Press, New York (2005)

24. Poetzsch-Heffter, A., Müller, P.: Logical foundations for typed object-oriented languages. In: Programming Concepts and Methods (PROCOMET), pp. 404-423 (1998)

25. Salcianu, A., Rinard, M.: Purity and Side Effect Analysis for Java Programs. In: Cousot, R. (ed.) VMCAI 2005. LNCS, vol. 3385, pp. 199-215. Springer, Heidelberg (2005)

26. Stevenson, D.E., Phillips, A.T.: Implementing object equivalence in Java using the template method design pattern. SIGCSE Bulletin 35(1), 278-282 (2003) 